Bangladesh J. Plant Taxon. 20(1): 1-8, 2013 (June)

(C) 2013 Bangladesh Association of Plant Taxonomists

\title{
THREE NEW SPECIES OF ELATOSTEMA J.R. FORST. \& G. FORST. (URTICACEAE) IN LIMESTONE CAVES FROM GUANGXI AND GUIZHOU, CHINA
}

\author{
Yi-Gang Wei, Fang Wen, Long-Fei Fu ${ }^{1}$ and Wen-Tsai Wang ${ }^{2 *}$ \\ Guangxi Institute of Botany, Guangxi Zhuang Autonomous Region and Chinese Academy of \\ Sciences, Guilin 541006, China
}

Keywords: Elatostema; New species; China.

\begin{abstract}
Three new species of Elatostema J.R. Forst. \& G. Forst. from Guangxi and Guizhou, China are described and illustrated. They are E. atrostriatum W.T. Wang \& Y.G. Wei, which is most similar to E. stewardii Merr.; E. jingxiense W.T. Wang \& Y.G. Wei, which is most similar to E. malacotrichum W.T. Wang \& Y.G. Wei, and E. schizodiscum W.T. Wang \& Y.G. Wei, which is most similar to E. angulaticaule W.T. Wang \& Y.G. Wei.
\end{abstract}

\section{Introduction}

Elatostema J.R. Forst. \& G. Forst. is a specialized genus for adapting to humid and dark habitats, e.g. dense forests, deep gorges and caves. It is one of the biggest genera in the family Urticaceae comprising at least 500 species all over the world, and mainly occur in tropical and subtropical Asia, Africa and islands of Oceania (Chen et al., 2003). Accompanying with the further field investigations on South and South-west China, more and more new taxa of this genus were discovered and published, including new series, Series Albopilosoides Q. Lin \& L.D. Duan (Lin and Duan, 2008), Series Neriifolia W.T. Wang \& Zeng Y. Wu (Wu et al., 2012), nearly 50 new species (Duan, 2010; Lin et al., 2011; Wang, 2010a, b, 2011a, b, 2012; Wei et al., 2011; Wei and Wang, 2011a, b; Wu et al., 2011a, b, 2012) and 4 new varieties (Wang, 2010b, 2012; Wu et al., 2011a). Bi et al. (2011) described new taxa of Elatostema from Thailand and India. Recently three unknown specimens belonging to the genus Elatostema were collected from Guangxi and Guizhou. After critical examination of these specimens and carefully consulting relevant literature, they were identified as new species.

1. Elatostema atrostriatum W.T. Wang \& Y.G. Wei sp. nov.

(Fig. 1).

Type: CHINA. Guangxi: Jiayou village, Lingyun County, 1 April 2009, Y.G. Wei 103 (Holotype: PE, Isotype: IBK).

Diagnosis: Species nova haec est similis E. stewardii Merr., quod foliis majoribus, apice cuspidatis, captitulis pistillis receptacula majorbus, bracteis pistillatis triangulatibus, dense puberulis, apice corniculatis, non striatis differt.

Herbs perennial, terrestrial. Stems c. 3 clumping, 100-135 $\times 1-2 \mathrm{~mm}$, erect, simple, glabrous. Stipules subulate, 1-7 $\times 0.1-0.3 \mathrm{~mm}$. Leaves distichous, alternate, short petioled, petioles 1-4 mm long, glabrous; leaf blades 28-105 $\times 10-34 \mathrm{~mm}$, oblique, narrowly obovate, leathery, nerves pinnate, broader-half lateral nerves $3-6,30-70^{\circ}$ to the midrib, narrower-half lateral nerves $4-6,30-$ $70^{\circ}$ to the midrib; upper surface cystoliths dense, conspicuous or inconspicuous, rod-shaped,

*Corresponding author. Email: wentsaiwang@yeah.net.

${ }^{1}$ College of Life Sciences, Guangxi Normal University, 541004, Guilin, China. 
${ }^{2}$ State Key Laboratory of Systematic and Evolutionary Botany, Institute of Botany, The Chinese Academy of Sciences, Cn-100093 Beijing, China.

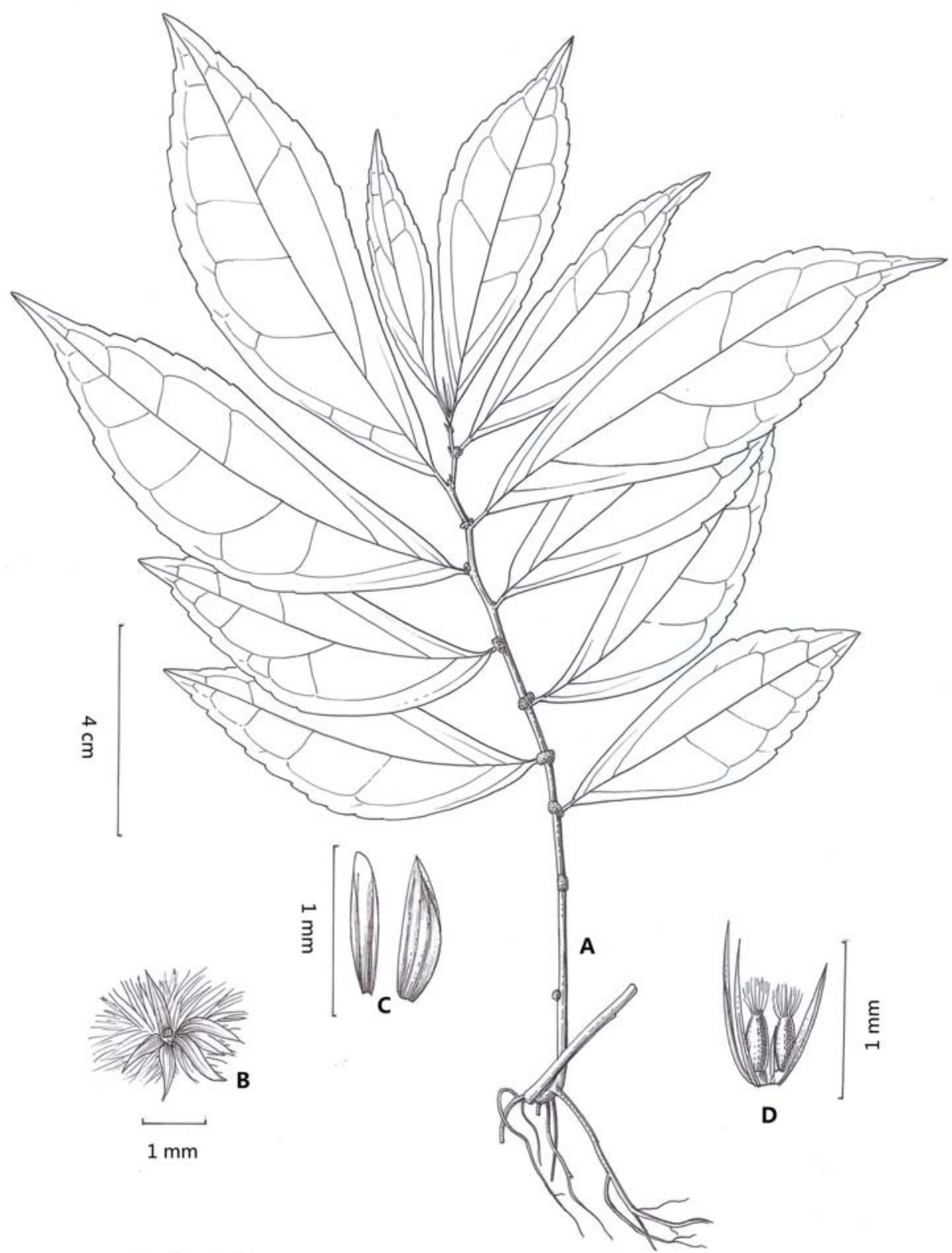

Fig. 1. Elatostema atrostriatum W.T. Wang \& Y.G. Wei: A. flowering stem; B. pistillate capitulum; C. two pistillatae bracts; D. three pistillate bracteoles and two pistillate flower (Drawn by Y.B. Sun from holotype).

0.1-0.25 mm long, lower surface cystoliths absent; base asymmetrical, broader-half rounded and narrower-half cuneate, margin entire or denticulate, basal $\leq 1 / 2$ entire, apex acuminate or blunt; 
Staminate capitulum not seen. Pistillate capitulum 1-3 axillary, sessile; receptacle inconspicuous; subtended by marginal bracts, bracts c. 5 , equal, membranous, semi-transparent, narrowly ovate or broadly linear, $0.8-1.0 \times 0.15-0.4 \mathrm{~mm}$, glabrous, with 2-3 black-brown longitudinal stripes; bracteoles numerous, dense, membranous, semitransparent, narrowly linear, $0.6-1.0 \mathrm{~mm}$ long, glabrous, with 1 black-brown longitudinal stripe. Pistillate flower short peduncled, tepal inconspicuous; pistil c. $0.7 \mathrm{~mm}$ long; ovary narrowly ellipsoid, $0.4 \mathrm{~mm}$ long; stigma $0.3 \mathrm{~mm}$ long. Infructescence not seen.

Habitat: Limestone cave, 20 individuals.

Etymology: Elatostema atrostriatum is named after pistillate bracts with 2-3 black-brown longitudinal stripes.

Vernacular name: Heiwen louticao (China).

Note: Elatostema atrostriatum is morphologically similar to E. stewardii Merr. but differs in leaves, pistillate receptacle, bracts and bracteoles (Table 1).

Table 1. Morphological comparison between Elatostema atrostriatum and E. stewardii

\begin{tabular}{lll}
\hline Characters & E. atrostriatum & E. stewardii \\
\hline Leaves & $\begin{array}{l}28-105 \times 10-34 \mathrm{~mm} \text {, apex acuminate or } \\
\text { blunt }\end{array}$ & $70-125 \times 28-45 \mathrm{~mm}$, cuspidate \\
Pistillate receptacle & $\begin{array}{l}\text { small, inconspicuous } \\
\text { Pistillate bracts }\end{array}$ & $\begin{array}{l}\text { narrowly ovate or broadly linear, glabrous, } \\
\text { not corniculate with 2-3 black-brown }\end{array}$ \\
$\begin{array}{l}\text { longitudinal stripes } \\
\text { glabrous, with 1 black-brown longitudinal } \\
\text { stripe }\end{array}$ & $\begin{array}{l}\text { triangular, densely pubescent, } \\
\text { corniculate with no stripes }\end{array}$ \\
Pistillate bracteoles & $\begin{array}{l}\text { margin densely pubescent with no } \\
\text { stripes }\end{array}$ \\
\hline
\end{tabular}

2. Elatostema jingxiense W.T. Wang \& Y.G. Wei sp. nov.

(Fig. 2).

Type: CHINA. Guangxi: Jingxi, Dizhou, limestone cave, 22 March 2009, Wei Yigang g067 (Holotype: PE, Isotype: IBK).

Diagnosis: Species nova haec est similis E. malacotrico W.T. Wang \& Y.G. Wei, quod plantis totis siccitate nigrescentibus, cauli staminati foliis normaliter evolutis eis cauli pistillati majoribus cystolithis densis praeditis, involucri staminati bracteis paucioribus ca. 8 depresse orbicularibus dorso puberulis distinguitur.

Herbs perennial, terrestrial, dioecious. Male plant: stems $150-500 \times 2-3 \mathrm{~mm}$, erect, dark brown when fresh, simple, densely puberulent, the hairs c. 0.1 mm long, 3-4 densely degraded microphyll at the top of the stem, underneath staminate capitulum bearing at naked nodes. Stipules lanceolate-linear, 3-4 mm long, glabrous. Leaves distichous, alternate, sessile or short petioled; leaf blades 12-14 $\times 4-16 \mathrm{~mm}$, chartaceous, oblique ovoid, nerves pinnate, lateral nerves 4 or 6 paired, $45-60^{\circ}$ to the midrib, upper surface sparsely hispid, hairs c. $0.5 \mathrm{~mm}$ long, weakly curved, appressed, cystoliths absent, lower surface nerves densely hispid, hairs c. $0.5 \mathrm{~mm}$ long, weakly curved, cystoliths absent; base asymmetric, broader-half oblique, broadly cuneate, narrower-half cuneate; margin dentate, apex acuminate, acumen entire. Female plant: stems $450-550 \times$ c. $5 \mathrm{~mm}$, erect, green when fresh, simple, densely puberulous, hairs c. $0.1 \mathrm{~mm}$ long; leaves distichous, alternate, short petioled, petiole 4-20 $\mathrm{mm}$ long; leaf blades $50-160 \times 35-65 \mathrm{~mm}$, chartaceous, oblique oblong or elliptic, nerves pinnate, lateral nerves 5 or 6 paired, $45-60^{\circ}$ to the midrib, upper surface sparsely hispid, hairs c. $0.5 \mathrm{~mm}$ long, weakly curved, appressed, cystoliths absent, lower 
surface nerves densely hispid, hairs c. $1 \mathrm{~mm}$ long, weakly curved, cystoliths absent; base asymmetric, broader-half rounded, narrower-half obliquely cuneate; margin dentate, broader-half

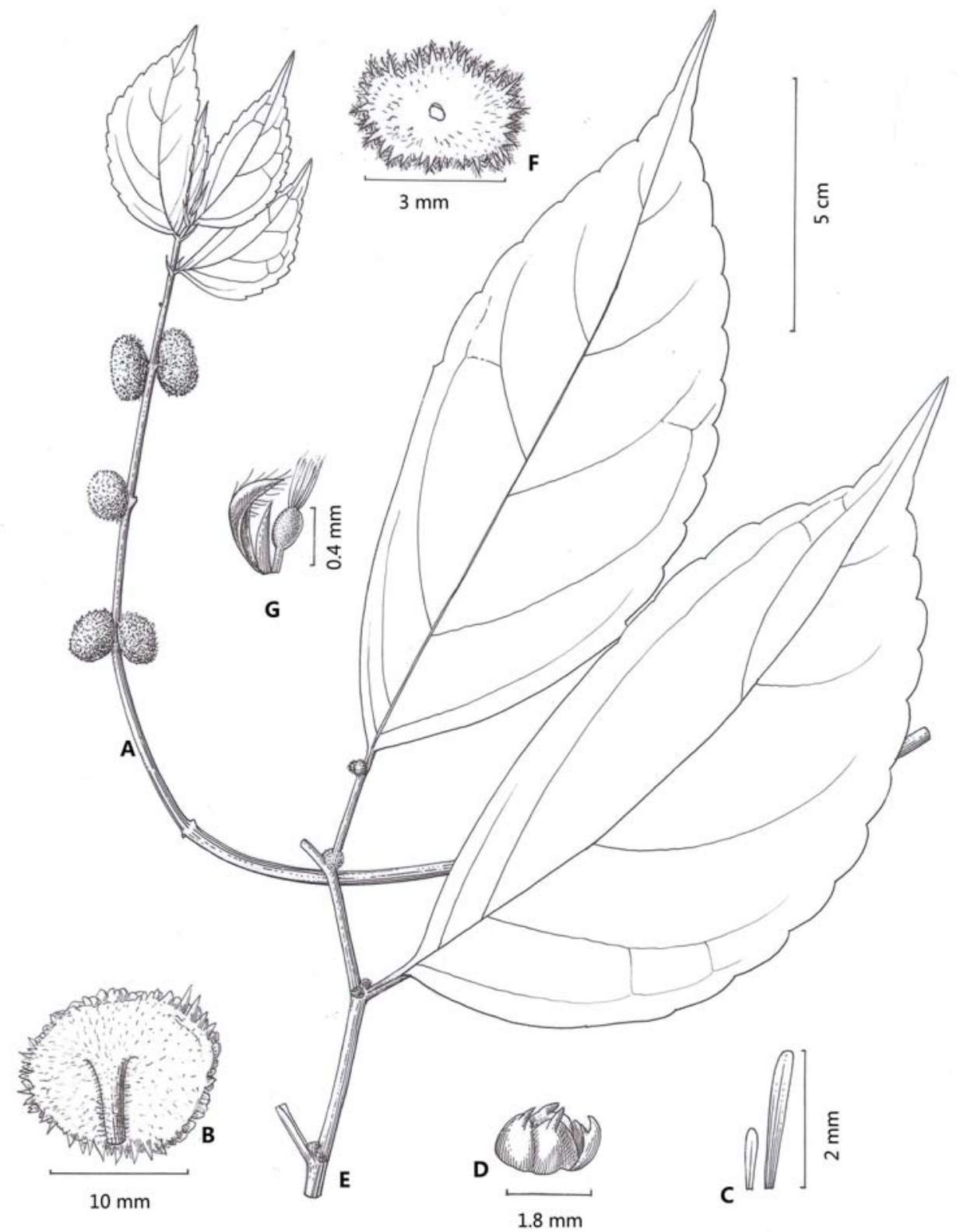

Fig. 2. Elatostema jingxiense W.T. Wang \& Y.G. Wei: A. staminate stem; B. staminate capitulum; C. staminate bracteoles; D. staminate flower; E. upper part of pistillate stem; F. pistillate capitulum; G. two pistillate bracteoles and pistillate flower (Drawn by Y.B. Sun from holotype). 
basal $\leq 1 / 3$ entire, narrower-half basal $\leq 1 / 2$ entire, apex acuminate, acumen entire. Staminate capitulum solitary or didymous, axillary, short pedunculate, c. $6 \mathrm{~mm}$ long, puberulent; receptacle subround, c. $10 \mathrm{~mm}$ in diam., puberulent, subtended by marginal bracts, the bracts c. 15 , membranous, triangular, c. $1 \mathrm{~mm}$ long, glabrous; bracteoles dense, membranous, semi-transparent, linear, 0.8-2.0 mm long, glabrous, with 1-3 brown stripes. Staminate flowers short peduncled, tepals 5 , broadly ovate, c. $1 \mathrm{~mm}$ long, subapical appendage c. $0.4 \mathrm{~mm}$ long, corniculate, glabrous. Pistillate capitulum didymous, axillary, sessile; receptacle elliptic, c. $3.0 \times 2.5 \mathrm{~mm}$, puberulent, subtended by marginal bracts, bracts c. 25 , narrowly triangular or linear, $0.3-0.5 \mathrm{~mm}$ long, puberulent or sometimes glabrous; bracteoles numerous, dense, membranous, cymbiform or linear-lanceolate, $0.5-0.8 \mathrm{~mm}$ long, puberulent or glabrous. Pistillate flower short peduncled, tepals inconspicuous; pistil $0.65 \mathrm{~mm}$ long; ovary ellipsoidal, c. $0.25 \mathrm{~mm}$ long; stigma $0.4 \mathrm{~mm}$ long.

Habitat: Limestone cave, 20 individuals.

Etymology: Elatostema jingxiense is named after the type locality near Jingxi County.

Vernacular name: Jingxi louticao (China).

Note: Elatostema jingxiense is morphologically similar to E. malacotrichum W.T. Wang \& Y.G. Wei but differs in colour of whole plant when dying, leaves of male stem and staminate bract (Table 2).

Table 2. Morphological comparison between Elatostema jingxiense and E. malacotrichum

\begin{tabular}{lll}
\hline Characters & E. jingxiense & E. malacotrichum \\
\hline $\begin{array}{l}\text { Whole plant turning } \\
\text { black while dying? }\end{array}$ & no & yes \\
Leaves of male stem & $\begin{array}{l}\text { much smaller than those of pistillate } \\
\text { stem and lacking cystoliths }\end{array}$ & $\begin{array}{l}\text { larger than those of pistillate stem and } \\
\text { bearing numerous dense cystoliths }\end{array}$ \\
Staminate bracts & c. 15, triangular and glabrous & $\begin{array}{l}\text { c. 8, depressed-orbicular and } \\
\text { puberulous abaxially }\end{array}$ \\
\hline
\end{tabular}

3. Elatostema schizodiscum W.T. Wang, \& Y.G. Wei sp. nov.

(Fig. 3).

Type: CHINA. Guizhou Province, Anlong County, Dushan town, limestone cave, 29 March 2010, F. Wen 1036, (Holotype: PE, Isotype: IBK).

Diagnosis: Species nova haec est similis E. angulaticauli W.T. Wang \& Y.G. Wei, quod foliis eorum nervis secundariis 6-8-jugis et eorum cystolithis minoribus, 0.05-0.1 mm longis, capituli pistillati receptaculo indiviso, floribus pistillatis ad receptaculum totum dense crescentibus, stigmatibus subglobosis recedit.

Herbs perennial, terrestrial. Stems c. $350 \times 4 \mathrm{~mm}$, decumbent, dark green, lower part drying 4-sulcate, distal part cystoliths dense, rod-shaped, 0.1-0.4 mm long, branched, glabrous. Stipules caducous. Leaves distichous, alternate, short petioled, petioles $2 \mathrm{~mm}$ long, glabrous; leaf blades $60-150 \times 30-35 \mathrm{~mm}$, oblique elliptic or oblique ovoid, chartaceous, nerves pinnate, lateral nerves 6-8 pairs, $45-60^{\circ}$ to the midrib, above sunken, beneath bulged out, conspicuous, upper surface sparsely hispid, hairs c. $1 \mathrm{~mm}$ long, weakly curved, cystoliths small, dense, rod-shaped or punctiform, 0.05-0.1 mm long, lower surface glabrous, cystoliths absent; base asymmetrical, broader-half broadly cuneate or oblique rounded and narrower-half cuneate; margin serrate, the apex of serrate blunt or rounded; apex long acuminate or acuminate, acumen entire. Staminate inflorescence not seen. Pistillate capitulum didymous, axillary, c. $5 \mathrm{~mm}$ wide, sessile; receptacle white, 2-partite, glabrous, lobes oblong or subquadrate, c. $2 \mathrm{~mm}$ long, undivided or 3-partite, apex 
bearing flowers dense, glabrous, subtended by bracts, bracts 3, white, broadly triangular, c. $1 \mathrm{~mm}$ long, glabrous, bracteoles white, narrow, c. $0.7 \mathrm{~mm}$ long, glabrous. Pistillate peduncle white, c. 0.7 $\mathrm{mm}$ long, glabrous, tepals c. 2, equal, narrowly linear, c. $0.3 \mathrm{~mm}$ long, glabrous; ovary elliptic, c. $0.22 \mathrm{~mm}$ long, stigma subglobose. Achene brown, narrowly ovate, c. $0.7 \times 0.3 \mathrm{~mm}$, with 4 longitudinal ribbs; staminodium c. 3 , white, narrowly ovate, c. $0.3 \mathrm{~mm}$ long, glabrous.

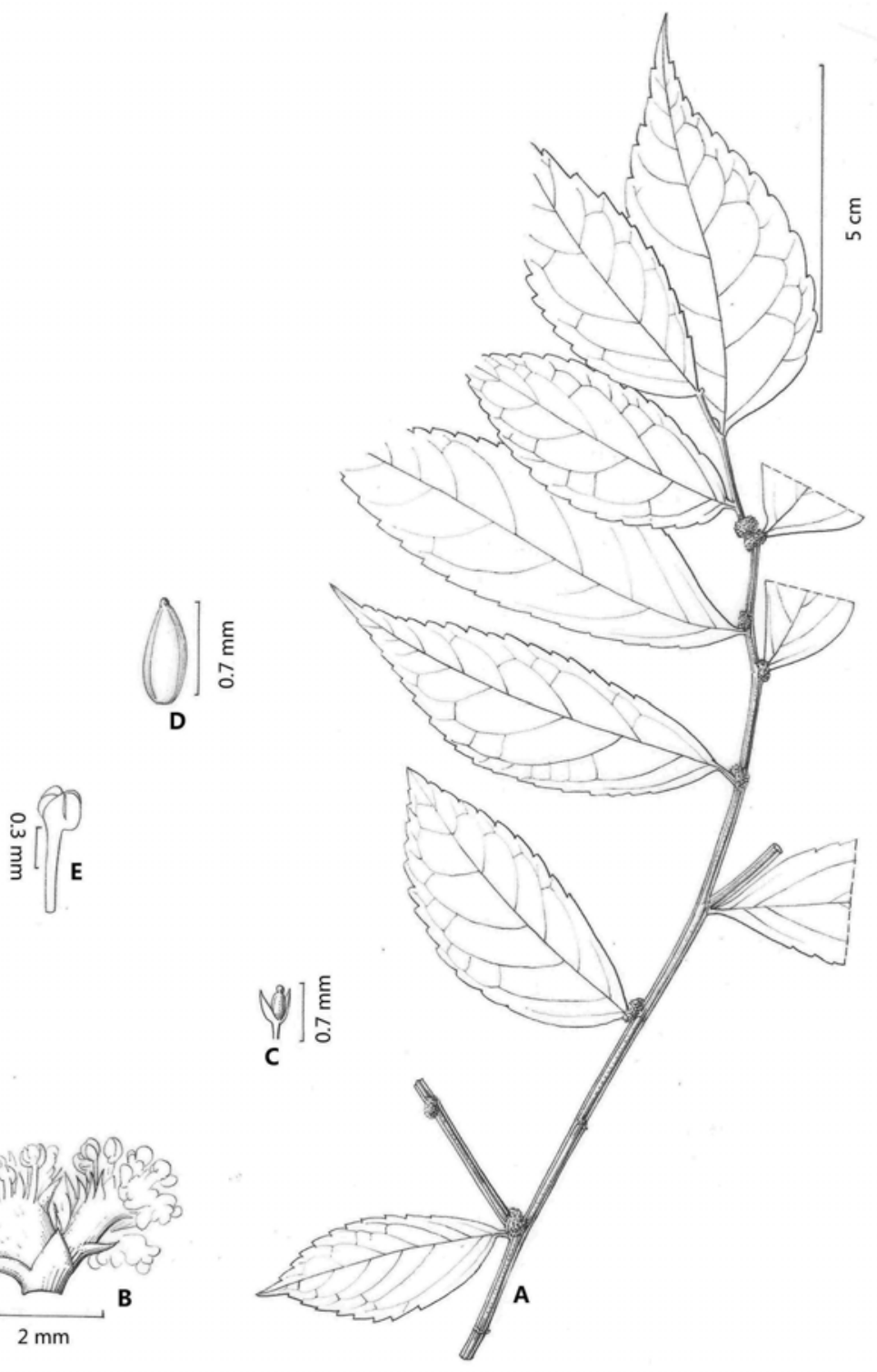

Fig. 3. Elatostema schizodiscum W.T. Wang \& Y.G. Wei: A. flowering pistillate stem; B. pistillate capitulum; C. pistillate bracteoles and pistillate flower; D. achene; E. fruiting pedicel and staminodes (Drawn by Y.B. Sun from holotype). 
Habitat: Limestone cave, 35 individuals.

Etymology: Elatostema schizodiscum is named after receptacle 2-parted.

Vernacular name: Lietuo louticao (China).

Note: Elatostema schizodiscum is morphologically similar to E. angulaticaule W.T. Wang \& Y.G. Wei but differs in leaves, cystoliths, pistillate receptacle and stigma (Table 3).

Table 3. Morphological comparison between Elatostema schizodiscum and E. angulaticaule

\begin{tabular}{lll}
\hline Characters & E. schizodiscum & E. angulaticaule \\
\hline Leaves & lateral nerves 6-8 pairs & lateral nerves 4-6 pairs \\
Cystoliths & $0.05-0.1 \mathrm{~mm}$ long & $0.1-0.3 \mathrm{~mm}$ long \\
Pistillate receptacle & 2-parted, flowers densely growing on & $\begin{array}{l}\text { undivided, flowers growing on } \\
\text { the whole receptacle }\end{array}$ \\
Stigma & apex of receptacle & \begin{tabular}{l} 
penicillate \\
\hline
\end{tabular} \\
\hline
\end{tabular}

\section{Acknowledgements}

We thank Mr. Ying-Bao Sun for the drawing, and Prof. Fa-Nan Wei for help. We also like to acknowledge the support of the National Natural Science Foundation of China (Grant No. 3116039), the Science Research Foundation of Guangxi Institute of Botany (Guizhiye11001), and the Science Research Foundation of Guangxi Institute of Botany (No. 10004) supporting the fieldwork.

\section{References}

Bi, H.Y., Yang, Z.R. and Lin, Q. 2011. New taxa of Elatostema (Urticaceae) from Thailand and India. Bangladesh J. Plant Taxon. 18(2): 149-152.

Chen, C.J., Lin, Q., Friis, I., Wilmot-Dear, C.M. and Monro, A.K. 2003. Urticaceae. In: Wu, Z. and Raven, P.H. (Ed.), Flora of China, Vol. 5. Science Press, Bejing, pp. 76-189.

Duan, L.D. 2010. Elatostema cataractum (Urticaceae), a new species from Guizhou Province, China. Ann. Bot. Fenn. 47: 229-232.

Lin, Q and Duan, L.D. 2008. Two new species and a new series of Elatostema (Urticaceae) from China, Bot. J. Linn. Soc. 158: 674-680.

Lin, Q., Shui, Y.M. and Duan, L.D. 2011. Elatostema oppositum (Urticaceae), a new species from Yunnan, China. Novon 21(2): 212-215.

Wang, W.T. 2010a. New taxa of Pellionia and Elatostema (Urticaceae) from China. Guihaia 30: 1-12.

Wang, W.T. 2010b. Notes on the genus Elatostema (Urticaceae). Guihaia 30(6): 713-727.

Wang, W.T. 2011a. Two new species of Elatostema (Urticaceaa) from Southeastern Yunnan. Guihaia 31(2): 143-147.

Wang, W.T. 2011b. Six new species of Elatostema (Urticaceae) from Gaoligong Shan, Yunnan. Plant Divers. Resour. 33: 145-156.

Wang, W.T. 2012. Four new species and one variety of Elatostema (Urticaceae) from China. Plant Divers. Resour. 34(2): 137-144.

Wei, Y.G., Monro, A.K. and Wang, W.T. 2011. Additions to the Flora of China: seven new species of Elatostema (Urticaceae) from the karst landscapes of Guangxi and Yunnan. Phytotaxa 29: 1-27.

Wei, Y.G. 2009. Notes on Elatostema J. R. et G. Forst (Urticeceae) from Guangxi. Guihaia 29(6): 711-718.

Wei, Y.G. and Wang, W.T. 2011a. Elatostema xanthotrichum and E. bamaense (Urticaceae), two new species from Guangxi, China. Ann. Bot. Fenn. 48: 93-95. 
Wei, Y.G. and Wang, W.T. 2011b. Elatostema recurviramum (Urticaceae), a new cave-dwelling species from Guangxi, China. Novon 21(2): 281-284.

Wu, Z.Y., Wang, W.T., Wang, H. and Li, D.Z. 2011a. Elatostema densistriolatum sp. nov., E. latistipulum sp. nov. and E. cyrtandrifolium var. hirsutum var. nov. (Urticaceae) from southwest China. Nord. J. Bot. 29: $227-232$.

Wu, Z.Y., Wang, W.T., Wang, H. and Li, D.Z. 2011b. Two new species of Elatostema (Urticaceae) from southeast Yunnan, China. PhytoKeys 7: 57-62.

Wu, Z.Y., Li, D.Z., Wang, H. and Wang, W.T. 2012. One new series with its only new species of Elatostema (Urticaceae) from Southeast Yunnan, China. Plant Divers. Resour. 34(2): 151-154.

(Manuscript received on 11 October 2012; revised on 20 March 2013) 